BULL. AUSTRAL. MATH. SOC.

\title{
Pseudo-distributive near-rings
}

\section{Henry E. Heatherly and Steve Ligh}

\begin{abstract}
In the study of the theory of rings, matrix rings, group rings, algebras, and so on, play a very important role. However, the analogous systems may not exist in the theory of near-rings. Recently Ligh obtained a necessary and sufficient condition for the set of $n \times n$ matrices with entries from a near-ring to be a near-ring. This opens the door for the study of other structures such as group near-rings, algebras, and so on. In this paper we initiate a study of the basic properties of pseudo-distributive near-rings, which is exactly the class of near-rings needed to carry out the construction of matrix near-rings, group near-rings, polynomials with near-ring coefficients, and so on.
\end{abstract}

\section{Introduction}

In the study of the theory of rings, matrix rings, group rings, algebras, and so on, play a very important role. However, the analogous systems may not exist in the theory of near-rings. In his dissertation [1] Beidleman considered the system $M_{n}(R)$ of all $n \times n$ matrices with entries from a near-ring $R$. He showed that if $R$ is a near-ring with identity and $M_{n}(R), n>I$, is a near-ring, then $R$ is a ring. Recently it was shown in [6] that $M_{n}(R)$ is a near-ring if and only if $R$ is an n-distributive near-ring. This opens the door for the study of other structures such as group near-rings, algebras, and so on. The purpose of this paper is to initiate a study of the basic properties of pseudo-distributive near-rings, which is exactly the class of near-rings needed to carry out the construction of matrix near-rings; group near-rings,

Received 20 February 1975. 
polynomials with near-ring coefficients, and so on.

\section{Definitions and examples}

DEFINITION 1. A near-ring $R$ is called $n$-distributive, $n$ a positive integer, if for each $a, b, c, d, r, a_{i}$, and $b_{i}$ in $R$,

(i) $a b+c d=c d+a b$, and

(ii) $\left(\sum a_{i} b_{i}\right)_{r}=\sum a_{i} b_{i}^{r}, i=1,2, \ldots, n$.

DEFINITION 2. A near-ring $R$ is called pseudo-distributive if it is $n$-distributive for each positive integer $n$.

It can easily be shown that a distributive near-ring is $n$-distributive for each $n$, hence, is a pseudo-distributive near-ring, while a distributively generated near-ring that is also pseudo-distributive must be distributive. We shall furnish some examples of pseudodistributive near-rings which are not distributive to illustrate the importance and abundance of the class of pseudo-distributive near-rings.

EXAMPLE 1. The near-rings $R$ given in $[3,2.1$, \#3, 2.2, \#16] have the property that $(R,+)$ is abelian and yet $R$ is not a ring. Recall that if $R$ is distributively generated, then $(R,+)$ is not abelian unless $R$ is a ring.

EXAMPLE 2. The near-ring given in $[3,2.2$, \#22], it is noteworthy to remark, is 3-distributive, but not 2 or 4-distributive. However, $0 x$ is not always zero.

REMARK. We shall assume throughout the rest of the paper that all near-rings $R$ considered have the property that $0 x=0$ for each $x$ in $R$. Thus if $R$ is $n$-distributive, then $R$ is m-distributive for each $m<n$.

EXAMPLE 3 [6]. ${ }^{\prime} M_{n}(R)$ is a near-ring if and only if $\dot{R}$ is n-distributive.

EXAMPLE 4. Group near-ring. Let $G$ be any group (written multiplicatively) and $N^{*}$ a near-ring. Let $N G$ be the set of all mappings from $G$ into $N$ which have finite support. 'Define addition pointwise and multiplication via $(t)_{\alpha} * \beta=\sum(g)_{\alpha} \cdot\left(g^{-1} t\right) \beta, g \in G, \alpha, \beta \in N G$, and 
$t \in G$. In general multiplication will not be well-defined, but will depend on the order of the elements in the sum. However, if $N$ is a pseudo-distributive near-ring, then $N G$ is also a pseudo-distributive near-ring. Conversely, if $N G$ is a near-ring and the order of $G$ is $k$, then $N$ is $k$-distributive. Hence it follows that $N$ is pseudodistributive if $G$ is an infinite group.

- EXAMPLE 5. Formal power series and polynomials. For an arbitrary near-ring $N$ define the formal power series $F N$ > over $N$ in the usual fashion, that is, each element $\left\langle a_{i}\right\rangle$ can be considered as a mapping from the set of non-negative integers into $N, i \rightarrow a_{i}$. Define addition pointwise and multiplication as the usual "Cauchy product":

$\left\langle a_{i}\right\rangle\left\langle b_{j}\right\rangle=\left\langle c_{n}\right\rangle$, where $c_{n}=\sum a_{i} b_{j}, i+j=n$. Then $F\langle N\rangle$ is a near-ring if and only if $N$ is pseudo-distributive. The subset of $F(N)$ of all functions of finite support, the polynomials over $N$, is a subnearring of $F\langle N\rangle$, when $F\langle N\rangle$ is a near-ring.

EXAMPLE 6. Gaussian near-ring. For an arbitrary near-ring $N$ define $N(i)$ to be the system composed of the group $(N,+) \oplus(N,+)$ together with "complex" or "gaussian" multiplication:

$$
(a, b)(c, d)=(a c-b d, a d+b c) \text {. }
$$

Then $N(i)$ is a near-ring if and only if $N$ is 2-distributive.

\section{Basic properties}

In this section we consider some elementary facts about pseudodistributive near-rings. It is worthwhile to mention that the class of pseudo-distributive near-rings is closed under direct products, epimorphic images, and subnear-rings; hence it is an equationally definable class (a variety). In this sense the class of pseudo-distributive near-rings is a better generalization of rings or distributive near-rings than is the class of distributively generated near-rings, which is not a variety, since a subnear-ring of a distributively generated near-ring need not be distributively generated [4]. Unfortunately the variety of pseudodistributive near-rings has many of the same limitations that the variety of distributive near-rings has as shown by the following result. 
THEOREM 1. Let $V$ be the variety of pseudo-distributive near-rings and let $V(p)$ be the class of all pseudo-distributive near-rings with property $p$. If $p$ is any one of the following properties, then $V(p)$. is $R(p)$, the class of rings with property $p$ :

(i) there exists a (Zeft, right, two-sided) identity;

(ii) there exists a left cancellable element;

(iii) every element is regular;

(iv) every element is an idempotent;

(v) every element is the sum of products;

(vi) there are no non-zero nilpotent ideals.

Proof. It is known that any distributive near-ring with any of the properties $(i)-(v i)$ must be a ring. It is easy to see that a pseudodistributive near-ring with any of the properties $(i)-(v)$ is distributive. The next theorem shows that a pseudo-distributive near-ring with property (vi) is a ring as well as setting up machinery needed in the sequel.

THEOREM 2. Let $R$ be a pseudo-distributive near-ring. Then

(i) the set $A=\{x \in R: R x=0\}$ is an ideal of $R$ and $R / A$ is a ring.

Thus the commutator subgroup $R^{\prime}$ of $R$ is a subset of $A$.

(ii) If $R$ is not a ring, then $A \neq 0$.

(iii) If $R$ has a pight identity, then $R$ is a ring.

(iv) The set $B=\{r \in R:(x+y) r=x p+y r, x, y \in R\}$ is a subnear-ring of $R$ and $A \subseteq B$. Also $(B,+)$ is $a$ normal subgroup of $(R,+)$.

Proof. (ii) If $(R,+)$ is abelian, then there is an $x \neq 0$ such that $x$ is not right distributive. Hence there are $w, z$ in $R$ such that $a=[(w+z) x-w x-z x] \neq 0$. It is easy to show that $a \in A$.

If $(R,+)$ is not abelian and $R$ is distributive, then $R^{\prime} \neq 0$ and $R^{\prime} \subseteq A$. If $R$ is not distributive, there is an $a \neq 0$ in $R$ such that $a \in A$ by the above argument.

The other parts follow from the definition of a pseudo-distributive 
near-ring and straightforward calculations.

If $R$ is a simple pseudo-distributive near-ring, then $A=R$ and $R^{2}=(0)$ or $A=(0)$ and $R$ is a ring. So simple pseudo-distributive near-rings are distributive and hence the direct sum or product of simple pseudo-distributive near-rings is a distributive near-ring with summands being either rings or zero multiplication near-rings.

The structure of a ring $R$ in which $(R,+)$ is a simple group is well-known; namely, $R$ is either the zero multiplication ring on $Z_{p}$ or $R$ is the finite field $\mathrm{GF}(p)$. Hence, if $N$ is a pseudo-distributive near-ring and $(N,+)$ is simple, then either $N$ is the zero multiplication near-ring on $(N,+)$ or $N$ is isomorphic to $\mathrm{GF}(p)$ for some prime $p$. Recall from Example 1 that there are pseudo-distributive near-rings which are not rings nor zero multiplication near-rings yet have $z_{n}$ as an additive group.

Note that if $R$ is a pseudo-distributive near-ring and $(R,+)$ is a perfect group (that is, $R^{\prime}=R$ ), then $R^{\prime}=A=R$ and $R$ is a zero multiplication near-ring. In contrast, the near-ring generated by the inner automorphisms on a finite non-abelian simple group $G$ is a nontrivial distributively generated near-ring whose additive group is the direct sum of copies of $G$ (and hence is perfect).

It is well known that the only ring that can be defined on a torsion divisible group is the zero ring. Now we consider pseudo-distributive near-rings defined on $Z\left(p^{\infty}\right)$.

THEOREM 3. Let $R$ be a pseudo-distributive near-ring and $(R,+) \cong Z\left(p^{\infty}\right)$. Then $R$ is the zero ring.

Proof. If every element is right distributive then $R$ is a ring and hence a zero ring. Suppose there is an element $w \neq 0$ that is not right distributive. As in the proof of (ii) of Theorem 2, there is an $a \neq 0$ in $R$ such that $a \in A$. Since $A$ is an ideal and $R / A$ is a ring, $(R / A,+) \cong Z\left(p^{\infty}\right)$. Let $x \in R$ and $B=\{x y: y \in R\} ; B$ is a homomorphic image of $R$ and hence divisible. But $B \subseteq A$ since $(x+A)(y+A)=A$. Since $A$ is finite, it follows that $B=0$. Thus $R$ has the zero multiplication.

It is well known that if $R$ is a ring with a finite number of proper 
subrings, then $R$ is finite. The situation for near-rings is unsettled, though it has been shown [2] to be affirmative for certain classes of nearrings. Recently, the above ring problem was investigated [5] from a different angle, by determining the structure of rings all of whose proper subrings are finite. Now we consider the case where $R$ is a pseudodistributive near-ring.

THEOREM 4. Let $R$ be an infinite pseudo-distributive near-ming in which each proper subnear-ring is finite. Then $R$ is one of the following:

(i) $R^{2}=0$ and $(R,+)$ is non-abelian;

(ii) $R^{2}=0$ and $R=Z\left(p^{\infty}\right)$ for some prime $p$;

(iii) $R=\bigcup_{n=0}^{\infty} \mathrm{GF}\left(p^{q^{n}}\right)$ for some primes $p$ and $q$.

Proof. Case 1. Suppose $R^{2}=0$. If $(R,+)$ is not abelian, then this is the well-known unsolved problem in group theory. If $(R,+)$ is abelian, then each proper subgroup is a subnear-ring, hence finite, and it is well known that $(R,+) \cong Z\left(p^{\infty}\right)$ for some prime $p$.

Case 2. Suppose $R^{2} \neq 0$. Thus there is an $x \neq 0$ in $R$ such that $x R \neq 0$. It is easy to see that both $x R$ and $A(x)=\{r \in R: x \dot{r}=0\}$ are subnear-rings of $R$. If $x R$ is finite, then $A(x)$ is infinite and hence $A(x)=R$, a contradiction. Thus $x R$ is infinite and $x R=R$. Now every element is right distributive and thus $R$ is a distributive near-ring. Also it can easily be shown that $R x=R$. Suppose $R^{\prime} \neq 0$ and let $r^{\prime} \in R^{\prime}$. There is a $y$ in $R$ such that $x y=r^{\prime}$. Let $e \in R$ such that $e x=x$. But $x y=r^{\prime}$ implies $e x y=e p^{\prime}=0$ and $x y=r^{\prime}=0$, a contradiction. Thus $R^{\prime}=0$ and $R$ is a ring and now the conclusion follows from the result in ring theory [5].

Right and anti-right distributive elements play an important role in the study of near-rings. (An element $x$ is anti-right distributive if and only if $(a+b) x=b x+a x$ for each $a, b$.$) In general, they do not form$ a subnear-ring. The following result gives a necessary and sufficient condition for them to be a subnear-ring. We shall omit the proof. 
THEOREM 5. Let $R$ be a near-ring. Then the set $T$ of right and anti-right distributive elements forns a subnear-ming if and only if $a r_{1}+b r_{2}=b r_{2}+a r_{1}$ for all $r_{1}, r_{2} \in T$ and $a, b \in R$.

Observe that in a pseudo-distributive near-ring $T$ is indeed a subnear-ring.

The next result shows how one can construct a pseudo-distributive near-ring from any given near-ring. The proof follows from direct calculation.

THEOREM 6. Let $R$ be a near-ring and let

$$
\begin{array}{r}
S(R)=\left\{x \in R: a x+b c=b c+a x,\left(\sum \dot{a}_{i} b_{i}\right) x=\sum a_{i} b x, i=1,2, \ldots, n,\right. \\
\left.a, b, c, a_{i}, b_{i} \in R\right\} .
\end{array}
$$

Then $S(R)$ is a pseudo-distributive subnear-ring of $R$.

Now we can examine $S(R)$ for certain classes of $R$.

THEOREM 7. Let $R$ be a near-field. Then $S(R)$ is a division ring.

Proof. Since the set $W$ of right distributive elements of $R$ is a division ring, we wish to show $W=S(R)$. Clearly $W \subseteq S(R)$. Since $S(R)$ is pseudo-distributive, hence $S(R)$ is 2-distributive. Let $x \neq 0$ be in $S(R)$ and $a, b$ be arbitrary elements of $R$. Then $x(a+b) x=(x a+x b) x=x a x+x b x=x(a x+b x)$. Since $x$ has a multiplicative inverse, it follows that $(a+b) x=a x+b x$. Thus $S(R) \subseteq W$ and the proof is complete.

Let $G$ be a group. We adopt the following notations for our next result:

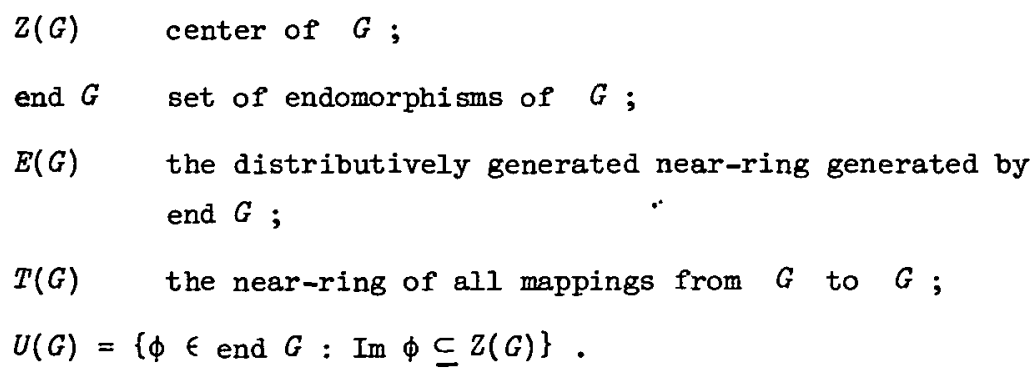

THEOREM 8. Let $G$ be any group. Then 
(i) $S(T(G))=U(G)$ and $U(G)$ is a ring,

(ii) $U(G) \subseteq S(E(G))$ and $S(E(G))$ is a ring.

Proof. To obtain $(i)$, let $\phi \in S(T(G))$. Since $T(G)$ has an identity $I$, for each $\alpha, \beta \in T(G)$,

$$
(\alpha+\beta) \phi=(\alpha I+\beta I) \phi=\alpha I \phi+\beta I \phi=\alpha \phi+\beta \phi .
$$

Hence $\phi$ is right distributive and it follows that $\phi \in$ end $G$. Suppose $x, y \in G$. Then there are $\alpha, \beta \in T(G)$ and $g \in G$ such that $g \alpha=x$ and $g \beta=y$. Hence $g(\alpha \phi+\beta I)=g(\beta I+\alpha \phi)$. It follows that $x \phi+y=y+x \phi$ and $\phi \in U(G)$. Similarly $U(G) \subseteq S(T(G))$. The fact that $U(G)$ and $S(E(G))$ are rings follows from the fact that both $T(G)$ and $E(G)$ have an identity.

Finally we remark that many of the results in this paper remain valid if one assumes $R$ only to be 2-distributive instead of pseudodistributive.

\section{References}

[1] James C. Beidleman, "On near-rings and near-ring modules", (Doctoral Dissertation, Pennsylvania State University, 1964).

[2] Howard Bell and Steve Ligh, "On finiteness conditions for near rings", Publ. Math. Debrecen (to appear).

[3] James R. Clay, "The near-rings on groups of low order", Math. 2. 104 (1968), 364-371.

[4] Henry Edward Heatherly, "Embedding of near-rings", (Doctoral Dissertation, Texas A\&M University, 1968).

[5] Thomas J. Laffey, "Infinite rings with all proper subrings finite", Amer. Math. Monthly 81 (1974), 270-272.

[6] Steve Ligh, "A note on matrix near rings", J. London Math. Soc. (to appear).

Department of Mathematics, Coll lege of Liberal Arts, University of Southwestern Louisiana, Lafayette, Louisiana, USA. 